\title{
SAR Image Data Compression Using a Tree-Structured Wavelet Transform
}

\author{
Zhaohui Zeng and Ian G. Cumming
}

\begin{abstract}
SAR image compression is very important in reducing the costs of data storage and transmission in relatively slow channels. In this paper, we propose a compression scheme driven by texture analysis, homogeneity mapping and speckle noise reduction within the wavelet framework. The image compressibility and interpretability are improved by incorporating speckle reduction into the compression scheme. We begin with the classical set partitioning in hierarchical trees (SPIHT) wavelet compression scheme, and modify it to control the amount of speckle reduction, applying different encoding schemes to homogeneous and nonhomogeneous areas of the scene. The results compare favorably with the conventional SPIHT wavelet and the JPEG compression methods.
\end{abstract}

Index Terms-Image texture, synthetic aperture radar (SAR), SAR image data compression, speckle reduction, set partitioning in hierarchical trees (SPIHT), wavelets.

\section{INTRODUCTION}

$\mathbf{S}$ YNTHETIC aperture radar (SAR) images represent an important source of information for earth scientists. However, while the volume of data collected is increasing rapidly, the ability to transmit it to the ground, or to store it, is not increasing as fast. Also, while storage densities on archiving media are improving with technological developments, our ability to generate new data is increasing even faster. Thus, there is a strong interest in developing data encoding and decoding algorithms that can obtain higher compression ratios while keeping image quality to an acceptable level.

There are some special characteristics of SAR imagery that affect the design of an image compression algorithm. The first is the speckle phenomena, which results from the coherent radiation and processing. A fully developed speckle pattern appears chaotic and unordered and severely degrades the quality of SAR images. The second is that there is detailed texture information as well as large homogeneous regions in SAR images. This makes it natural to consider a way to reduce coding bits within homogeneous regions of terrain. The third is that the very high dynamic range of SAR images is unlike that of image data from other Earth-imaging sensors, such as optical sensors. These differences mean that encoding/decoding algorithms de-

Manuscript received September 2, 1999; revised June 1, 2000. This work was supported in part by MacDonald Dettwiler, Richmond, BC, Canada, the Natural Sciences and Engineering Research Council of Canada, the BC Advanced Systems Institute, and the Science Council of British Columbia, Canada.

Z. Zeng was with the Department of Electrical and Computer Engineering, The University of British Columbia, Vancouver, BC, Canada V6T 1Z4. He is now with STMicroelectronics, Inc., Nepean, ON, Canada K2H 8R6.

I. G. Cumming is with DLR/DFD, Oberpfaffenhofen, Germany, on leave from the Department of Electrical and Computer Engineering, The University of British Columbia, Vancouver, BC, Canada V6T 1 Z4.

Publisher Item Identifier S 0196-2892(01)02087-3. signed for optical data may not be optimized or even appropriate for SAR data.

Wavelet transforms have received significant attention because their multiresolution decomposition allows efficient image analysis [1], [2]. They have been used in analysis, noise reduction and data compression of SAR images [3] as well as multi-spectral images [4]. Using the discrete wavelet transform (DWT), the procedures of terrain segmentation, speckle noise reduction, and data compression can be efficiently combined in a single process of decomposition and reconstruction [5]. In this work, we develop an algorithm using tree-structured texture analysis, soft-thresholding speckle reduction, and quadtree homogeneous decomposition using a modified set partitioning in hierarchical trees (SPIHT) encoding scheme. Our results are compared with the conventional JPEG and SPIHT wavelet transform schemes and show that our method is very promising for compression and image interpretation, particularly as texture information is preserved better.

Before we describe our new algorithm in Sections V and VI and the experimental results in Section VII, we introduce three important and somewhat independent concepts in the next three sections: texture analysis, homogeneous set partitioning, and speckle reduction.

\section{TeXture AnAlysis With TREe-STRUCTURED WAVELET TRANSFORM}

Using the DWT, every coefficient at a given scale can be related to a set of coefficients at the next finer scale of similar orientation. The coefficient at the coarse scale is called the parent, and all coefficients corresponding to the same spatial location at the next finer scale of similar orientation are called the children. For a given parent, the set of all coefficients at all finer scales of similar orientation corresponding to the same location are called descentants.

The traditional pyramid-type wavelet transform recursively decomposes subsignals into low frequency channels. However, since the most significant information of texture often appears in the middle frequency channels, recursive decomposition just into the lower frequency region may not be optimum for SAR images, which contain a great deal texture information. Thus, an appropriate way to perform the wavelet transform for texture is to detect the frequency channels with significant texture energy, and decompose them further.

Here we approach this problem by analyzing SAR images by the tree-structured wavelet transform used in [6]. The key point in our approach is that we no longer simply apply the decomposition to the low frequency subimages only. Instead, decomposition can be applied to the output of any filter $h_{L L}, h_{L H}, h_{H L}$, 
or $h_{H H}$, which is found to contain significant information ${ }^{1}$. The test for significance of texture can be described by the following steps.

1) Decompose an image using a two-dimensional (2-D) twoscale wavelet transform into four subimages, which can be viewed as the parent and children nodes in a tree, as shown in Fig. 1.

2) Calculate the average energy $e$ of each decomposed subimage $x(m, n)$ (the children nodes)

$$
e=\frac{1}{M N} \sum_{m=1}^{M} \sum_{n=1}^{N}|x(m, n)|^{2} .
$$

3) If the energy of a subimage is significantly smaller than others of the same level, we stop the decomposition in this region since it contains less information. This step can be achieved by comparing the energy $e$ with the largest energy value $e_{\max }$ found among all the subimages in the same scale (the same level in Fig. 1) in different orientations. That is, if $e<C e_{\max }$, stop decomposing this region, where $C$ is a constant less than 1 .

4) If this test shows the texture energy of a subimage to be significant, we apply the above decomposition procedure to the subimage once again.

The LL subimage has the highest energy, so it is always decomposed up to a certain maximum level. The maximum decomposition level is determined by the texture analysis. It is useful to set an upper limit on the decomposition to avoid unnecessary computation. In our SAR images, we found that a limit of $16 \times 16$ pixels per subimage to be sufficient.

For the other subimages, $\mathrm{LH}, \mathrm{HL}$, and $\mathrm{HH}$, the condition $e<C e_{\max }$ is used to determine whether there is enough information content to decompose them as well. The parameter $C<1$ is adjusted to control the degree of the texture analysis. This decomposition method is analogous to computing the complete wavelet packet transform to a certain maximum level, then pruning the branches from top to bottom using the above energy threshold.

The texture measures are created during this procedure. The degree of texture is represented as a series of parameters $\left\{F_{1}, F_{2}, \cdots, F_{l}, \cdots\right\}$ corresponding to a set of frequency channels or subimages. $F_{l}$ is a constant between 0 and 1 , defined as

$$
F_{l}=\frac{K L}{\sigma_{l}}
$$

where $L$ is the decomposition level of the subimage, $\sigma_{l}$ is the standard deviation of the subimage, and $K$ is a compensation factor, which is kept constant for a given type of image (a given type of texture). In a frequency channel with more texture, $F_{l}$ is smaller. In a channel with less texture, $F_{l}$ is larger.

An example of a 3-level, tree-structured, wavelet decomposition of a $256 \times 256$ pixel textured image is shown in Fig. 1 .

\footnotetext{
${ }^{1}$ The DWT decomposition is applied with quadrature mirror filters, which separate (filter) the image into high and low frequency subimages at each stage. The nomenclature $h_{H L}$ refers to the subimage with the high-frequency $\mathrm{H}$ filter applied in the horizontal direction and the low-frequency L filter applied in the vertical direction. Applying $\mathrm{H}$ and $\mathrm{L}$ filters in each of the horizontal and vertical directions results in the image being separated into four subimages, each with one quarter of the pixels of the parent image.
}

This illustrates the energy and texture distribution provided by the wavelet decomposition. Note that in the level 2 nodes $(2,0)$, $(2,2)$, and $(2,12)$, there is more texture and higher energy, so the decomposition is carried to the next higher level ("high" is down in the figure).

\section{HOMOGENEITY MAP}

An image "map," which specifies the degree of homogeneity of image texture and features, can be helpful in achieving higher compression gain, because we can allocate fewer bits to homogeneous regions, while allocating more bits to those regions containing more detail and sharper features. Here we apply a very simple segmentation scheme based on quadtree decomposition of the image at the lowest scale.

Quadtree decomposition is an analysis technique that involves subdividing an image into blocks that are more homogeneous than the whole image itself. It starts by decomposing the whole image into four equal-sized blocks and then testing each block to see if it meets some criterion of homogeneity (e.g., if all the pixels in the block are within a specific dynamic range). If a block meets the homogeneity criterion, it is not divided any further. If it does not meet the criterion, it is subdivided again into four blocks, and the test criterion is applied to those blocks. This process is repeated iteratively until each block meets the criterion.

After this quadtree decomposition, we get two sets: a homogeneous set and a target set. The homogeneous set consists of the relatively homogeneous regions. Each homogeneous region is represented by the coordinates of the pixel at its top left corner and by the size of the region. The target set consists of those single-component regions, represented by their coordinates (a single-component region is a single pixel in the LL subimage). The test criterion we choose is to split a block if the maximum value of the block elements minus the minimum is greater than the threshold $t_{h}$

$$
t_{h}=\gamma \sigma_{h} \bar{x}(m, n)
$$

$$
\begin{array}{cl}
\begin{array}{cl}
\text { where } \\
\sigma_{h}
\end{array} & \begin{array}{l}
\text { standard deviation of the wavelet coefficients at the } \\
\text { highest frequency band in the diagonal direction; }
\end{array} \\
\bar{x}(m, n) & \begin{array}{l}
\text { average value of the coefficients of each block; } \\
\text { constant selected to give the threshold a reasonable } \\
\text { (and adjustable) level. }
\end{array}
\end{array}
$$

\section{SPECKLE REDUCTION}

As discussed in references such as [7], the speckle noise typically can be modeled as a multiplicative identical independent distribution (i.i.d.) Gaussian noise. Logarithmic transformation of a SAR image converts the multiplicative noise model to an additive noise model and has been used successfully in [3].

For a digitized SAR image, we define $y(j, k)$ as the gray level (the observed image magnitude) of the $(j, k)$ th pixel of the image. Hence, the pixel level of a SAR image can be written as

$$
y=x e
$$



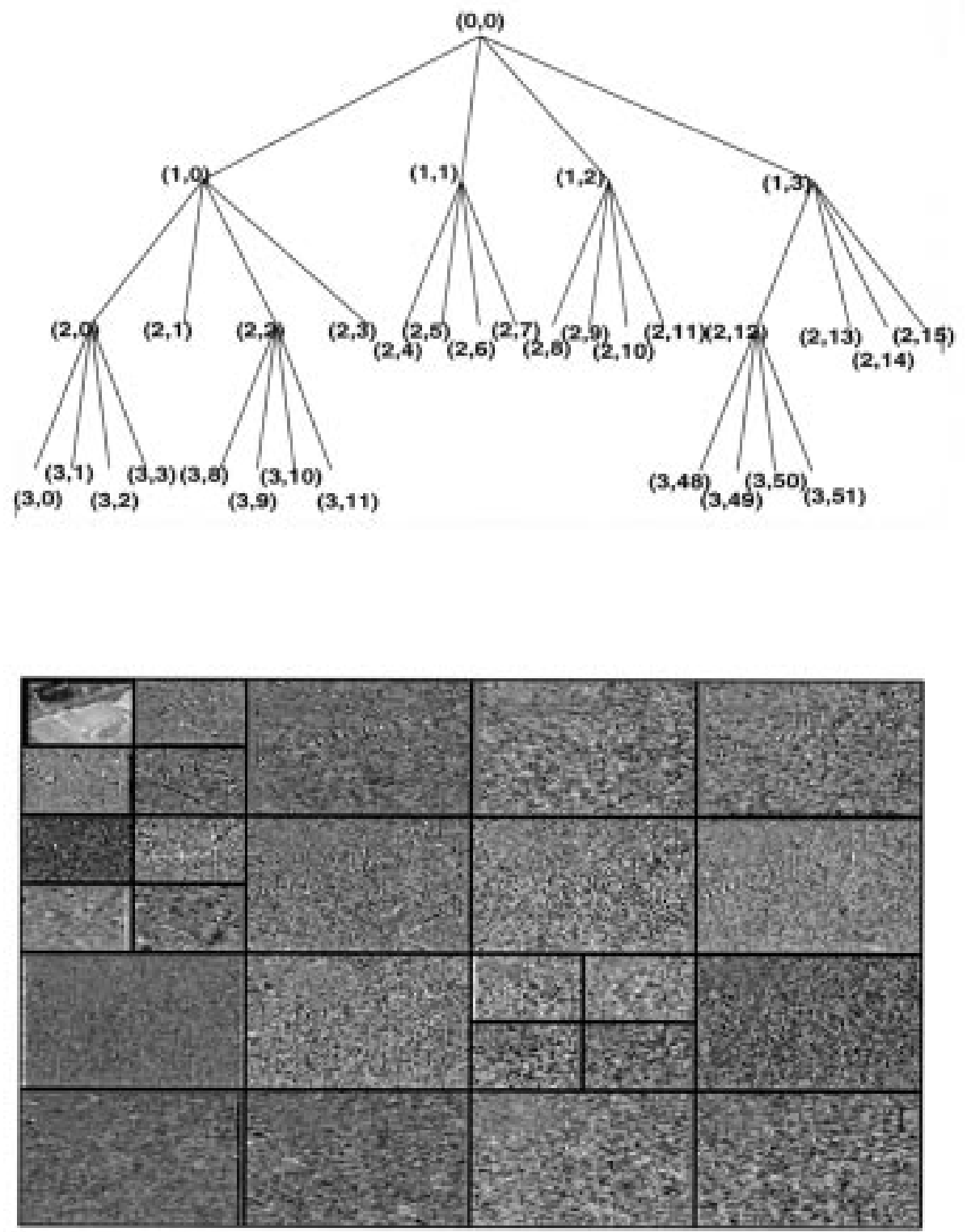

Fig. 1. Tree-structured wavelet decomposition of a single-look airborne radar image. Referring to the tree structure, the node $(0,0)$ represents the logarithm of the original image, and we refer to it as the "lowest" level. The top row of subimages correspond to nodes $(3,0),(3,1),(2,1),(2,4)$, and $(2,5)$ of the tree structure.

where $x$ is the desired radiometric information, and $e$ is the multiplicative speckle noise. Arsenault and April [7] showed that for logarithmically transformed SAR images, the speckle is approximately Gaussian additive noise

$$
\bar{y}=\bar{x}+\bar{e}
$$

where $\bar{y}=\ln (|y|), \bar{x}=\ln (|x|)$, and $\bar{e}=\ln (|e|)$. If $W$ is the multilevel DWT, then a multiresolution representation is given by the equation

$$
W \bar{y}=W \bar{x}+W \bar{e} \quad \text { or } \quad Y=X+E
$$

where $Y=W \bar{y}, X=W \bar{x}$, and $E=W \bar{e}$. The standard deviation of the noise in the multiresolution representation $\sigma$ is not known in advance and must be estimated from the data.

To reduce the contribution of the smallest coefficients that contain mainly noise, a soft threshold [8] is applied to all the wavelet coefficients except those of the lowest scale. A softthreshold operation involves subtracting $t_{l}$ sign $\{$ coeff $\}$ from each coefficient. This removes some of the speckle inherent in SAR imagery while preserving much of the detail, thereby increasing the image interpretability and the available compressibility. The value of the threshold $t_{l}$ is given by

$$
t_{l}=F_{l} \sigma_{h} \sqrt{2 \log \left(n_{l}\right)}
$$

where $n_{l}$ is the number of pixels in each frequency band, and $F_{l}$ is the texture parameter (2) of the corresponding frequency channel.

\section{MODIFIED SPIHT CODING SCHEME}

The embedded zero-tree wavelet coding (EZW) [1] is a very effective and computationally simple technique for image compression. The "set partitioning in hierarchical trees" method 


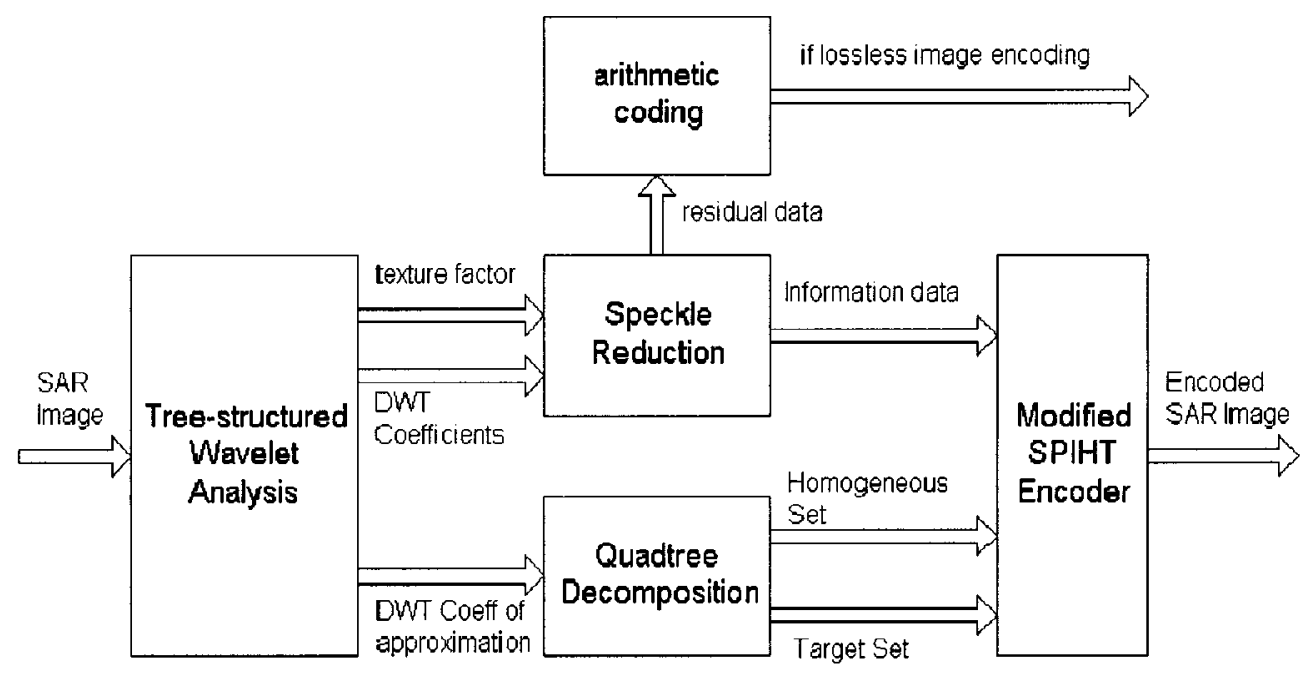

Fig. 2. Block diagram of the modified SPIHT coding scheme, incorporating speckle reduction. The quadtree decomposition to separate the homogeneous and target areas is applied only to the LL subimage of the lowest level, often referred to as the "approximation" subimage.

(SPIHT) [2] improves upon the performance of EZW based on three concepts:

1) partial ordering of the transformed image elements by magnitude, with transmission order determined by a subset partitioning algorithm that is duplicated at the decoder;

2) ordered bit plane transmission of refinement bits;

3) exploitation of the self-similarity of the image wavelet transform across different scales.

The SPIHT algorithm offers low complexity, embedded coding, and good performance, making it a suitable candidate for remotely sensed images [4]. In our work, we modify the SPIHT algorithm by adding soft-thresholding, homogeneous mapping, and the texture parameters $F_{l}$ to make it more suitable for compressing SAR images.

Normally, most of an image's energy is concentrated in the low frequency components. Consequently, the variance of the DWT coefficients decreases as we move from the highest to the lowest levels of the subband pyramid. Furthermore, it has been observed that there is a spatial self-similarity between subbands, and the coefficients are expected to be better magnitude-ordered if we move downward in the pyramid following the same spatial orientation. For instance, low-activity areas can be identified in the highest levels of the pyramid and can be followed to the lower levels at the same spatial locations.

A tree structure called a spatial orientation tree naturally defines the spatial relationship of the nodes on the hierarchical pyramid. Fig. 1 shows how our spatial orientation tree is defined in a pyramid constructed with recursive four-subband splitting. Each node of the tree corresponds to a pixel and is identified by the pixel coordinate. Its direct descendants (offspring) correspond to the pixels of the same spatial orientation in the next finer level of the pyramid. The tree is defined in such a way that each node has either no offspring (the leaves) or four offspring, which always form a group of $2 \times 2$ adjacent pixels. In Fig. 1, the lines lead down from the parent node to its four offspring. The pixels in the highest level of the pyramid (bottom row in the figure) are the rest roots and are also grouped in $2 \times 2$ sets of adjacent pixels.

A wavelet coefficient $Y$ is said to be insignificant with respect to a given threshold $T$ if $|Y|<T$ [2]. The zerotree structure is based on the hypothesis that if a wavelet coefficient at a coarse scale is insignificant with respect to a given threshold $T$, then all wavelet coefficients of the same orientation in the same spatial location at finer scales are likely to be insignificant with respect to $T$. In the progressive transmission mode, the $n$th bit of the wavelet coefficients is transmitted if $2^{n} \leq\left|c_{i, j}\right| \leq 2^{n+1}$. To make the relationship between magnitude comparisons and message bits clear, we use the following function to indicate the significance of a set of coordinates $\tau$ :

$$
S_{n}(\tau)= \begin{cases}1, & \text { if } \max \left\{\left|c_{i, j}\right|\right\} \geq 2^{n},(i, j) \in \tau \\ 0, & \text { otherwise. }\end{cases}
$$

Our method is different from the conventional EZW or SPIHT methods in the following ways.

1) Different parents may have the same children because the tree-structured wavelet transform allows the size of children to be smaller than that of the parent, which is unlike the pyramid structure used in the SPIHT method. For example, in Fig. 1, the coefficient at the scale branch $(3,2)$ has descendants at nodes $(3,8),(3,9),(3,10),(3,11),(2,8)$, $(2,9),(2,10)$, and $(2,11)$, as well as at other nodes ${ }^{2}$.

2) If the descendants have more than one parent, they are regarded as significant once they satisfy the condition of the threshold $T_{i}$ from any one of their parents. The descendants are insignificant only when they satisfy the condition of the thresholds $\left\{T_{1}, T_{2}, \cdots, T_{i}\right\}$ from all of their parents.

3) The condition (8) of $S_{n}$ is changed to be $\max \left\{\left|c_{i, j}\right|\right\} \geq$ $F_{l} 2^{n},(i, j) \in \tau$, where $F_{l}$ is the texture factor (2). In

\footnotetext{
${ }^{2}$ The first index is the decomposition level, the second index is the location within that level. The coordinate $(3,0)$ is the LL subimage at the highest level of decomposition, and $(0,0)$ is the original image (not shown in the image part of Fig. 1).
} 

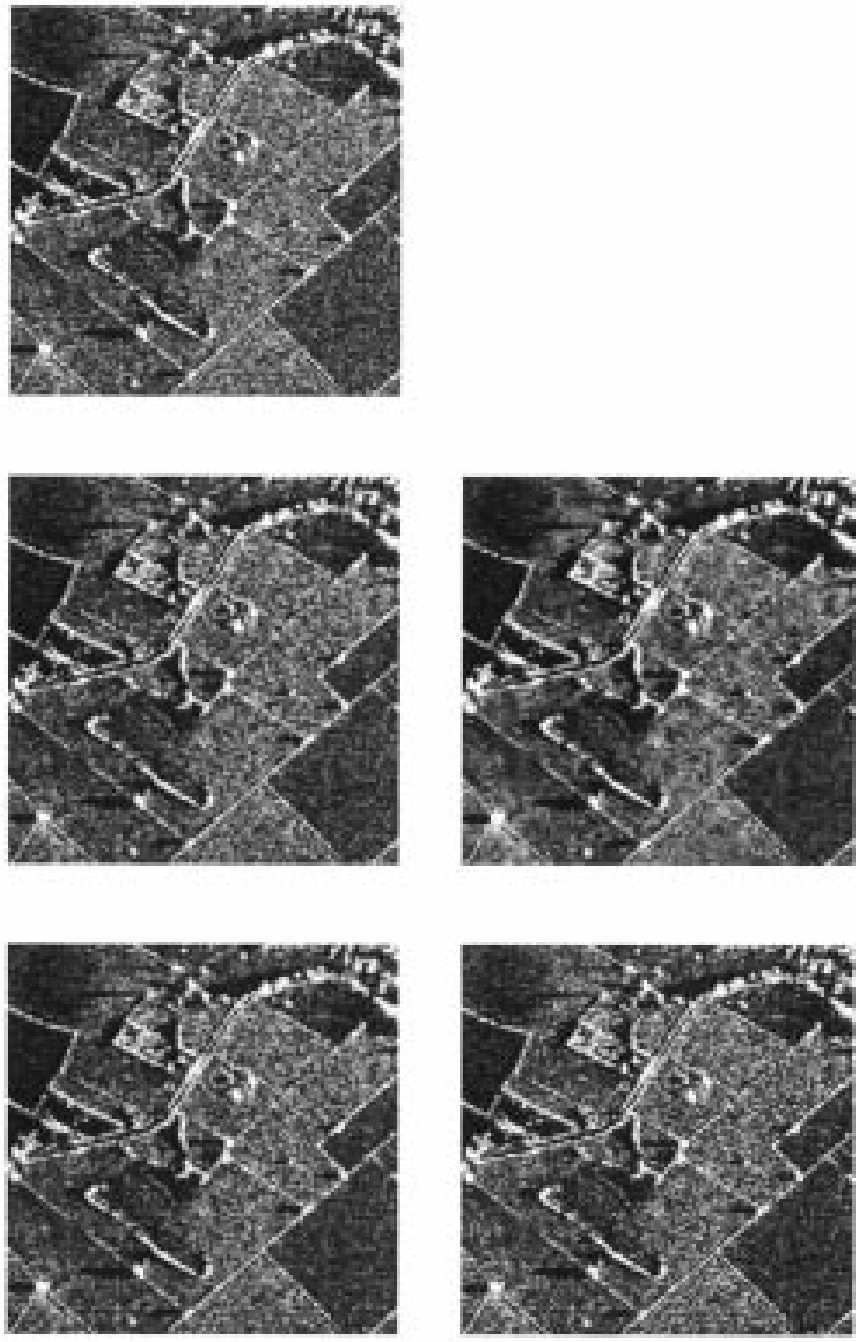

Fig. 3. Comparison of compression/filtering results at $1.0 \mathrm{bpp}$.

this way, texture information has the highest priority in bit allocation.

4) Two different coefficient sets, the homogeneous set and the target set, are encoded separately.

\section{New Encoding Scheme Including SPeCKLE REDUCTION}

Because the homogeneity map of the lowest scale has been created by the quadtree decomposition, we can improve the coding efficiency by encoding the homogeneity map (Section III) with more bits allocated to selected areas of greater detail. To further improve the coding efficiency, soft-thresholding (Section IV) is applied before the start of coding of other scales, reducing the number of coefficients to be encoded. If the user requires lossless coding, the residuals from soft-thresholding and coding are kept and losslessly compressed using arithmetic coding. However, these residuals consist mainly of speckle, so they are often ignored.

The new coding scheme is outlined in Fig. 2, where the separate steps of speckle reduction and quadtree homogeneity analysis can be seen. The scheme is applied after the logarithm has
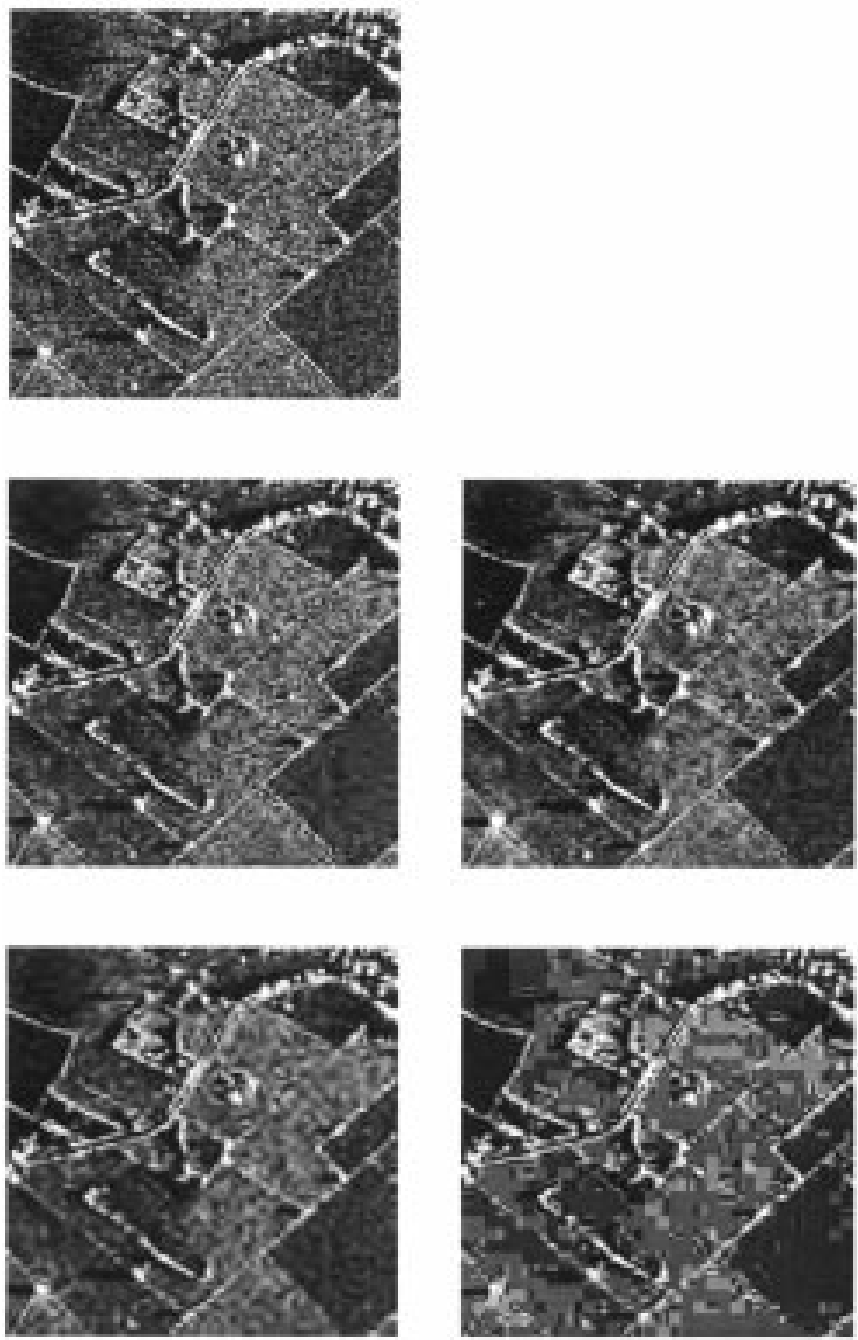

Fig. 4. Comparison of compression/filtering results at $0.2 \mathrm{bpp}$.

been taken of the data. The features of the new method are as follows.

- Control of speckle reduction: The degree of speckle reduction is flexible based on the requirements of the user. The speckle reduction step can be omitted if it is not required.

- Speckle reduction: Soft-thresholding is applied to all wavelet coefficients except the lowest scale ones. After quantization, the number of significant bits of the wavelet coefficients is reduced. The residuals are kept and transmitted if needed.

- Encoding the homogeneous set: For the components in the homogeneous list, we send the decoder the average value, the dynamic range, and the size of the homogeneous region, and then use this average value as the threshold to decide the significant coefficients at the next finer scale. Then we compute the difference between the value of every component in the homogeneous region and the average value. We quantize these differences and transmit them in bit-plane order.

- Tree-structured coding: The wavelet coefficients in the target list are quantized using the modified SPIHT algo- 
rithm. At every scale branch, three candidate encoding schemes are tested: no encoding, arithmetic coding, and run-length coding. The candidate with the best performance is chosen as the encoding scheme at this scale branch.

- Lossless residual coding: Efficient compression of the residuals is essential to the efficiency of the algorithm as a whole. These coefficients possess a rapidly decaying exponential distribution with a large percentage of zero coefficients. This skewed distribution makes arithmetic coding the best choice for lossless compression.

\section{EXPERIMENTAL RESULTS}

For our experiments, we chose a scene of a rural area surrounding the town of Stanwick in Bedfordshire in southeast England, shown in Fig. 3(a) [9]. The scene contains many urban and natural features that typically appear in high-resolution airborne SAR imagery. The scene is from the British Defense Research Agency (DRA) X-band radar, and has $3 \mathrm{~m}$ resolution, one look, and is stored in amplitude units. The data was originally in 32-bit floating point format, but we reduced it to 8 bits for ease of use in our data conversion and imaging software. We feel that this reduction has no impact on the qualitative nature of our results. In our experiments, Daubechies 4 (DB4) wavelet transforms (quadrature mirror filter pairs) are employed [10].

In order to assess the effectiveness of our compression algorithm on SAR imagery, three schemes are applied: our modified SPIHT (MSPIHT), conventional SPIHT, and JPEG. The MSPIHT scheme is used with and without speckle reduction. Quantitative measurements are used to evaluate the performance of the compression schemes. Because the potential of speckle noise reduction within SAR image compression is being investigated, the usual metrics of compression performance are not completely appropriate to our algorithm. We have used three types of compression quality measurements [11] as follows.

1) Peak Signal to Quantization Noise Ratio (PSQNR): defined as the ratio, in $\mathrm{dB}$, of the largest pixel value in the image to the mean squared error (MSE). The MSE measure gives the total encoding error between original and reconstructed images in an absolute sense.

2) Standard Deviation to Mean Ratio ( $\mathbf{s} / \mathbf{m})$ : is a suitable indication of image speckle when measured in homogeneous regions.

3) Target-to-clutter ratio (t/c): is the difference in magnitude (in $\mathrm{dB}$ ) between the target peak and the local clutter. It measures how a bright target stands out from the surrounding clutter.

The results of each of these four encoding methods are shown in Figs. 3, Fig. 4, and the quality assessment parameters are given in Tables I-III. From these results, we can see that wavelet methods (both MSPIHT and conventional SPIHT) outperform JPEG, particularly at high compression ratios. This can be observed in both the visual results and the numerical values. For MSPIHT, the results with speckle reduction are better than without speckle reduction at the same compression ratio, particularly when the compression ratio is high. More texture information is kept by the algorithm when speckle re-
TABLE I

COMPARISON OF TARget HeIGHT PRESERVATION AT 0.2 bp

\begin{tabular}{r|c}
\hline & $\mathrm{t} / \mathrm{c}(\mathrm{dB})$ \\
\hline Original Image & 41.7 \\
\hline De-noised Image & 42.0 \\
\hline MSPIHT without denoise & 31.8 \\
\hline MSPIHT with denoise & 34.1 \\
\hline Conventional SPIHT & 32.2 \\
\hline JPEG & unacceptable \\
\hline
\end{tabular}

TABLE II

PSQNR (dB) FOR RECONSTRUCTED SAR IMAGES

\begin{tabular}{r|c|c|c|c}
\hline & $2 \mathrm{bpp}$ & $1 \mathrm{bpp}$ & $0.5 \mathrm{bpp}$ & $0.2 \mathrm{bpp}$ \\
\hline MSPIHT without denoise & 40.2 & 39.3 & 36.5 & 33.8 \\
\hline MSPIHT with denoise & 42.3 & 41.5 & 38.2 & 35.4 \\
\hline Conventional SPIHT & 39.8 & 38.5 & 35.4 & 32.1 \\
\hline JPEG & 37.7 & 35.4 & 32.3 & 23.1 \\
\hline
\end{tabular}

TABLE III

COMPRESSION PERFORMANCE OF THE MSPIHT ALGORITHM, WITH AND Without SPECKLE NOISE REDUCTION

\begin{tabular}{c|c|c|c|c|c|c}
\hline & \multicolumn{3}{|c|}{ With noise reduction } & \multicolumn{3}{c}{ Without noise reduction } \\
\hline Bit rate & $1.0 \mathrm{bpp}$ & $0.5 \mathrm{bpp}$ & $0.1 \mathrm{bpp}$ & $1.0 \mathrm{bpp}$ & $0.5 \mathrm{bpp}$ & $0.1 \mathrm{bpp}$ \\
\hline $\mathrm{s} / \mathrm{m}$ & 0.144 & 0.138 & 0.129 & 0.232 & 0.213 & 0.201 \\
\hline $\mathrm{t} / \mathrm{c}$ & 35.8 & 35.7 & 33.5 & 33.8 & 33.4 & 32.3 \\
\hline PSQNR & 41.5 & 38.2 & 33.4 & 39.3 & 36.5 & 31.3 \\
\hline
\end{tabular}

duction is used. See for example, the fields in Fig. 4, including the shadows and structure in the fields.

It is clear that the speckle reduction itself results in some compression. Comparing both the visual and numerical results between MSPIHT with speckle reduction and without, we can see that the compressibility of the despeckled image is improved. For example, even at very high compression ratios (e.g., 0.2 bpp), the despeckled MSPIHT keeps most of important structural information of the original image, while MSPIHT without despeckling and conventional SPIHT does not. However, it is clear that some texture information has been lost at $0.2 \mathrm{bpp}$ even with the modified SPIHT scheme, so we do not recommend compression ratios this high when image interpretability is important. Similar results were obtained with RADARSAT-1 spaceborne data.

We see in Table I that the dynamic range of bright targets has been reduced by the compression scheme. While this loss may harm some applications, the target detectability is still good because the surrounding speckle is reduced. One of the advantages of wavelet-based compression schemes is that they reduce data dynamic range while retaining the essential texture and interpretability of the image.

\section{IMPLEMENTATION ISSUES}

In practice, SAR images are much larger than the $512 \times 512$ image we have used to obtain the results above. In order to com- 
press a larger image while retaining the computing efficiency of small images, we suggest one of the following two approaches.

The first is Method 1, in which we divide the large image into several smaller images, of a size that can be handled efficiently. Apply the proposed algorithm to each of them, using symmetrical or zero extension to reduce edge effects. While some edge effects may persist, they will be much less than DCT-based methods at the same compression ratio.

The second is Method 2, in which we apply the proposed tree-structured decomposition of this paper, but control the decomposition level at each scale and orientation based upon the texture analysis and the image application. Accepting a lower decomposition level improves efficiency with a relatively small accuracy penalty.

The encoding overhead of the modified SPIHT algorithm is slightly more than the standard SPIHT algorithm, because we also encode the labels of the target and homogeneous sets. Note that the tree structure does not have to be encoded, as it is only used to analyze the image to find our where the strong texture lies, which is used to control the speckle reduction and compression.

\section{CONCLUSIONS}

A SAR image compression algorithm is presented which combines texture analysis, speckle reduction and image compression, and accomplishes embedded coding. From the experiments, we found the following.

- The discrete wavelet transform has proven to be efficient in SAR image processing. The DWT allows us to analyze images from different bands and spatial orientations based on the image features. The advantages of DWT analysis are more applicable to SAR images than that to other remote sensing images because of the rich texture information contained in SAR imagery. The benefits of the DWT are particularly evident in speckle reduction and image compression.

- We have placed priority on the preservation of image texture by applying a higher level of decomposition where the texture is stronger and by the use of a texture factor in the soft-thresholding.

- Simultaneous speckle reduction and data compression can significantly improve the compressibility of SAR images. The effectiveness of this strategy has been shown in comparison with the same encoder without speckle reduction and with the conventional DWT compression algorithm (SPIHT).

- The relationship of wavelet coefficients at different frequency bands and spatial orientations can provide more efficient ways to represent the properties of SAR images and is worthy of future investigation.

\section{REFERENCES}

[1] J. Shapiro, "Embedded image coding using zerotrees of wavelet coefficients," IEEE Trans. Signal Processing, vol. 41, pp. 3445-3462, Dec. 1993.

[2] A. Said and W. Pearlman, "A new, fast, and efficient image codec based on set partitioning in hierarchical trees," IEEE Trans. Circuits Syst. Video Technol., vol. 6, pp. 243-250, June 1996.

[3] D. Wei, H. Guo, J. Odegard, M. Lang, and C. Burrus, "Simultaneous speckle reduction and data compression using best wavelet packeet bases with application to SAR based ATD/R," in SPIE Conference on Wavelet Applications, vol. 2491, Orlando, FL, Apr. 1995.

[4] P. L. Dragotti, G. Poggi, and A. R. P. Ragozini, "Compression of multispectral images by three-dimensional SPIHT algorithm," IEEE Trans. Geoscience and Remote Sensing, vol. 38, pp. 416-428, Jan. 2000.

[5] M. Vetterli and J. Kovacevic, Wavelets and Subband Coding. Englewood Cliffs, NJ: Prentice-Hall, 1995.

[6] T. Chang and C.-C. J. Kuo, "Texture analysis and classification with tree-structured wavelet transform," IEEE Trans. Image Processing, vol. 2, pp. 429-441, Oct. 1993.

[7] H. H. Arsenault and G. April, "Properties of speckle integrated with a finite aperture and logarithmic transformation," Opt. Soc. Amer., vol. 66, p. $1160,1976$.

[8] D. Donoho, "De-noising by soft-thresholding," IEEE Trans. Inform. Theory, vol. IT-41, pp. 613-627, May 1995.

[9] C. Oliver and S. Quegan, Understanding Synthetic Aperture Radar Images. London, U.K.: Artech House, 1998.

[10] I. Daubechies, "Time-frequency localization operators-A geometric phase space approach," IEEE Trans. Inform. Theory, vol. 34, pp. 605-612, 1988.

[11] M. Dutkiewicz and I. Cumming, "Evaluation of the effects of encoding on SAR data," Photogramm. Eng. Remote Sensing, vol. 60, pp. 895-904, July 1994.

Zhaohui Zeng received the B.Sc. in engineering physics from Tsinghua University, Beijing, China, in 1993 and the M.A.Sc degree in electrical and computer engineering from the University of British Columbia (UBC), Vancouver, BC, Canada, in 1999.

Following the graduate work in the Radar Remote Sensing Laboratory at UBC, he joined Nortel Networks and was involved in the development and implementation of digital signal processing algorithms applied in TDMA and CDMA wireless base stations. Currently, he is working in the Broadband Wireline Division, STMicrolectronics, Nepean, ON, Canada. His research interests include digital signal processing and wireless communication.

Ian G. Cumming received the B.Sc. in engineering physics from the University of Toronto, Toronto, ON, Canada, in 1961, and the Ph.D. in computing and automation from Imperial College, University of London, London, U.K., in 1968.

Following work in steel mill automation and sonar signal processing, he joined MacDonald Dettwiler and Associates, Richmond, BC, Canada, in 1977. Since that time, he has developed synthetic aperture radar signal processing algorithms and worked on systems for processing polarimetric and interferometric radar data, and for the compression of radar data. He has been involved in the algorithm design of the digital SAR processors delivered by MacDonald Dettwiler for SEASAT, SIR-B, ERS-1/2, J-ERS-1, and Radarsat, as well as several airborne radar systems. In 1993, he joined the Department of Electrical and Computer Engineering, University of British Columbia, Vancouver, where he holds the MacDonald Dettwiler/NSERC Industrial Research Chair in Radar Remote Sensing. The Radar Remote Sensing Laboratory supports a research staff of six engineers and students working in the fields of SAR processing, SAR data encoding, satellite SAR two-pass interferometry, airborne along-track interferometry, airborne polarimetric radar classification, and SAR Doppler estimation. He and his associates are principal investigators on the ERS Tandem, the Radarsat ADRO, and the SRTM/X-SAR Missions. 